solid tumours is the principal burden on the surgeon. The techniques for many forms of surgery practised on tumours today were developed in the second half of the nineteenth century after Lister's discovery of the use of antiseptics. There was no doubt that since that time tremendous improvements have been made in the survivals of some cancer patients subject to surgery but, nevertheless, Mr Burn saw that there is much room for improve. ment. Early diagnosis was without doubt the most useful aid to the surgeon; at present far too many cases of advanced cancer are operated upon.

\section{AGEING}

\section{New Interest}

from our Cell Physiology Correspondent Although familiarity undoubtedly breeds contempt, this cannot be the only reason for the indifferent attitude of many people towards research on ageing. Possibly the way in which the problem is casually dismissed arises from an appreciation of some of the technical difficulties involved and a reluctance to undertake experiments which may take years to complete.

Almost seven years ago a short cut to this problem was suggested, namely that of ageing "in vitro", but although the evidence for a relationship between the finite life span of human diploid fibroblasts in culture and ageing "in vivo" is now very strong, there still does not seem to be a closer understanding of the basic principles involved.

Two recent articles in Experimental Cell Research arouse optimism, however, and suggest that interest in this field may be picking up. Lima and Macieira-Coelho $(\mathbf{7 0}, 279$; 1972) have examined the changes associated with discontinuity in growth of chick embryo fibroblasts and they conclude that along with human and bovine diploid strains, these chick embryo cells may be used to study ageing in vitro: the cells can be passaged about thirty times every second day, so that the total life span is complete in less than three months, which is about half the time that human diploid fibroblasts take to die.

Apparently there are some differences between the ageing patterns of these chick fibroblasts and the human ones, for the time taken for chick cells to enter DNA synthesis does not change throughout the life of the cells and does not slow down even when the saturation density is declining. During the later passages, DNA synthesis is not followed by a doubling of the cell population, and Lima and Macieira-Coelho give two possible reasons. Like the ageing fibroblasts of other species, it is possible that the $G_{2}$ period is prolonged but it is also likely that the ageing cells may have an increased sensitivity to cell crowding and may detach from the glass. This last suggestion indicates that ageing in chick fibroblasts has not quite the same characteristics as those of human and bovine fibroblasts and is supported by the fact that increasing numbers of cells are found in the supernatants of ageing chick cultures.

Many similarities exist, however, for apart from the decline in growth rate, chick fibroblasts also show the characteristic increase in cell size during the later passages. No doubt the easiest explanation of this phenomenon is to assume that it is merely a reflexion of the reduced potential for cell division, but the increase in size in this instance is not associated with a decrease in the capacity for division; the significant decline in saturation density occurs at a much later passage. This dissociation of ageing parameters may seem to be difficult to explain but it does suggest that ageing in vitro is not the result of any single event but is rather the end process in a sequence of changes.

In the same issue (ibid., 436) Goldstein and Lin show that it is possible to rescue senescing cells, in this case human ones, by hybridization with hamster kidney cells. Although their results do not provide any positive evidence for the nature of ageing, one or two ideas on the subject lose their plausibility. The system obviously provides a convenient method for obtaining interspecific hybrids, for it is possible to select against both parental cells using 6-thioguanine resistant cells of the BHK line and senescent human fibroblasts, incapable of further cell division. Hybrid clones have been obtained which have been subcultured through more than a hundred cell divisions so that theories which are based on ideas of irreversible cross-linking of DNA, irreversible histone binding or dominant genetic ageing factors would appear to be an unlikely cause of ageing in vitro.

Goldstein contends that ageing is a quasi-differentiated event which is amenable to reprogramming, but he does not suggest any mechanism which might bring this about. Indeed, it would seem possible to interpret the results in favour of either of the two basic theories of ageing. Genetic reprogramming may well occur but it is also possible that accumulated faults in informational macromolecules may be diluted and "repaired" by the more accurate macromolecules originating from hamster kidney cells. Although it is difficult to think of a definitive experiment which might distinguish between these two possibilities, curiosity leads one to wonder what might result if two senescent cell cultures were hybridized.

\title{
Basis of Steroid Resistance
}

ONE of the great obstacles to the treatment by chemotherapy of people suffering from chronic diseases such as autoimmune disease and cancer is the tendency of the patient to develop resistance to the therapeutic agents. Presumably the population of target cells in the body is continually throwing up variants which are more resistant to the drugs being used than the rest of the population, and in the presence of the drugs such variants find themselves at a selective advantage and consequently come to predominate in the population.

The way in which somatic cell geneticists select drug resistant variant cells by passaging their cells in culture media containing a particular drug, the concentration of which is gradually increased during the selection procedure, may be a comparatively simple model of the situation faced by the chemotherapist. That at least is the contention of Rosenau, Baxter, Rousseau and Tomkins, whose analysis of the basis of resistance of cultivated mouse $1 \mathrm{ym}$ phoid cells to glucocorticoids is reported in Nature New Biology next Wednesday.

Glucocorticoids cause lymphoid cells to lyse and for that reason they are used to treat cancer and other diseases of the lymphoid system. Cultivated mouse lymphoid cells are also susceptible to lysis by these steroids and steroid resistant variants can easily be isolated. Tomkins and his colleagues have recently developed cell free systems which allow them to measure two steps in the interaction of lymphoid cells with glucocorticoids; namely, the initial step of the binding of the steroid to a cytoplasmic receptor and the second step, the interaction of the receptor-steroid complex with the nucleus of the target cell. Using dexamethasone as the challenge steroid they have compared the extent of its binding at these two steps in cell free systems obtained from dexamethasone sensitive and resistant cells.

Rosenau et al. find that the binding of the steroid by cytoplasmic extracts of resistant cells is significantly less than the binding observed with similar extracts from sensitive cells, and this is not the result of an inhibitor of binding in the resistant cell extracts. Nuclei of resistant cells bind the receptor-steroid complex almost as well as the nuclei of sensitive cells. It seems, therefore, that the resistance of cultivated mouse lymphoid cells to lysis by glucocorticoids results from a change at the cytoplasmic receptor level rather than at the nuclear receptor level. 